\section{a Results of Compliant Participation in Five Rounds of Fecal Immunochemical Test Screening for Colorectal Cancer}
Q21 Flavia Baldacchini,, Lauro Bucchi, ${ }^{*}$ Orietta Giuliani, ${ }^{*}$ Silvia Mancini, ${ }^{*}$ Alessandra Ravaioli, ${ }^{*}$ Rosa Vattiato, ${ }^{*}$ Paolo Giorgi Rossi, ${ }^{\ddagger}$ Cinzia Campari, ${ }^{\S}$ Debora Canuti, " Enza Di Felice," Francesca Mezzetti," Priscilla Sassoli de Bianchi, " Stefano Ferretti, ${ }^{\#}$ and Fabio Falcini, ${ }^{*, * *}$ on behalf of the Emilia-Romagna Region Workgroup for Colorectal Screening Evaluation

${ }^{*}$ Romagna Cancer Registry, Romagna Cancer Institute, Istituto Scientifico Romagnolo per lo Studio e la Cura dei Tumori, IRCCS, Meldola, Forli, Italy; ${ }^{\ddagger}$ Epidemiology Unit, ${ }^{\S}$ Cancer Screening Unit, Azienda Unità Sanitaria Locale, IRCCS di Reggio Emilia, Reggio Emilia, Italy; "Cancer Screening Unit, Local Health Authority, Rimini, Italy; "Department of Health, Regional Administration, Emilia-Romagna Region, Bologna, Italy; "University of Ferrara and Local Health Authority, Ferrara, Italy; ${ }^{* *}$ Cancer Prevention Unit, Local Health Authority, Forli, Italy

\section{Q10 BACKGROUND \& AIMS:}

We investigated the magnitude and temporal patterns of the decreasing trend in main performance measures of fecal immunochemical test (FIT) screening for colorectal cancer (CRC) observed in second and subsequent rounds.

METHODS:

RESULTS:

CONCLUSIONS:
We followed up 494,187 participants from the first round of a regional biennial FIT screening program in Italy (cut-off value for positivity, $20 \mu \mathrm{g}$ hemoglobin/g feces) for 5 total rounds (2005-2016). At each round, only compliant participants were eligible. Performance measures from the first, third, fourth, and fifth round were compared with those from the second round (the first incidence round) using rate ratios from multivariate Poisson regression models and relative risk ratios from multinomial logistic regression models.

Between the second and the third round, a significant $20 \%$ to $30 \%$ decrease was found in the proportion of men with a positive FIT result (from $5.2 \%$ to $4.3 \%$ ) and in detection rates of advanced adenoma (from 13.4 to 10.2 per 1000), CRC (from 1.7 to 1.4 per 1000), and advanced neoplasia (from 15.1 to 11.6 per 1000). Positive predictive values (PPVs) decreased by $10 \%$ or less between the second and third rounds. Detection rates and PPVs for adenoma stabilized by the fourth and fifth rounds. The PPVs for advanced adenoma, CRC, and advanced neoplasia decreased slightly in men and women by the fourth and fifth rounds. The detection rate of proximal colon cancer stabilized after the second round, whereas the detection rate of distal CRC decreased until the fourth round in men (from 0.7 to 0.3 per 1000), and the fifth round in women.

These findings support the notion that FIT screening prevents progression of a subset of advanced adenomas. Screening intensity could be modulated based on results from previous rounds, with a risk-based strategy. 
However, it is unclear whether the decrease continues for certain performance measures after the second round. This depends, first, on the varying design of the relevant studies. In general, the authors defined a screening round as a complete cycle of invitations, during which the whole target population was invited. ${ }^{1-5,8}$ Under this design, all participants in any given round were evaluated irrespective of their participation in previous rounds. For example, subjects who responded to an invitation after 2 refusals contributed to the results of the third screening round, although they were actually at their prevalence round. In 2 studies, conversely, only subjects with compliant participation to previous rounds were considered eligible. ${ }^{6,7}$ Subjects participating in the third round, for example, were eligible only if they also had participated in the first 2 rounds.

Second, most studies had a small size and covered limited observation times. Some studies considered only the first 2 rounds. ${ }^{2,5}$ In a study covering 6 rounds, the proportion of positive FIT results, the detection rate (DR) of advanced adenoma, and the PPV for advanced adenoma decreased between the first and the second round, and then stabilized. The DR of CRC, instead, continued to decrease, with a DR ratio of the sixth round to the first round as low as 0.18 . $^{7}$

Third, most previous studies did not make an allowance for the aging of the population, which is associated with increasing risk of disease. This explains, for example, the increase in the proportion of positive FIT results and the DR of advanced neoplasia that was observed at the fourth round of a screening trial. ${ }^{8}$

This article describes a large study of this issue, which aimed to evaluate the performance of compliant participation in the first 5 rounds of a biennial FIT screening program in Italy.

\section{Materials and Methods}

\section{Setting}

In the Emilia-Romagna Region (northern Italy), a population-based CRC screening program has been ongoing since March 2005. The target population includes the residents of both sexes ages 50 to 69 years ( $\mathrm{n}=1,037,532$ on January 1,2005$)$. The program is run at the health care district level $(\mathrm{n}=11)$ according to a standard protocol.

Every 2 years, eligible subjects are invited with a personal letter to perform a single-sample FIT. Most kits are distributed by public pharmacies and primary care facilities. ${ }^{9}$ Nonresponders to the invitation are mailed a reminder, usually within 6 months. The screening test is a latex agglutination test (OC-Sensor; Eiken Chemical Co, Tokyo, Japan) without dietary restrictions. The cut-off value for positivity is $20 \mu \mathrm{g}$ hemoglobin/g feces $(100$ ng hemoglobin/mL of buffer). Subjects are notified of negative FIT results by mail. Subjects with positive

\section{What You Need to Know}

\section{Background}

Little is known about the magnitude and temporal patterns of the decreasing trends in main performance measures of fecal immunochemical test (FIT) screening for colorectal cancer that have been observed in second and subsequent rounds.

\section{Findings}

Findings from the study support the concept that FIT screening prevents progression of a subset of advanced adenomas. Screening intensity could be modulated based on results from previous rounds, with a risk-based strategy.

\section{Implications for patient care}

FIT screening strategies for colorectal cancer might be designed specifically for each patient based on results from previous rounds of screening.

results are contacted by telephone, invited to attend a screening center, and referred for complete conventional colonoscopy under sedation. In the case of incomplete colonoscopy, patients are presented with the option of a 916 virtual colonoscopy. Patients with positive FIT results and a negative colonoscopic assessment are re-invited to a FIT screening 5 years later.

\section{Objectives and Design}

The study had a cohort design. The objectives were to contrast the performance measures of FIT screening at each of the first 5 rounds in a population of compliant participants with the average measures observed in the total screening population, and to investigate the decrease observed among compliant participants by comparing the third, fourth, and fifth round with the second round (ie, the first incidence round).

Regarding compliant participants, the performance measures were calculated cross-sectionally, for 5 rounds, in a cohort of subjects including all residents ages 50 to 69 years who were invited and had a FIT during the first round of the screening program. The dates of FITs varied between March 21, 2005, and December 31, 2016. A compliant participant was defined as one who had 2, 3, 4, or 5 consecutive FITs at standard intervals (ie, 2 y \pm 6 mo). The follow-up evaluation of each FIT ceased on the date of the following events, whichever came first: last regular FIT; receipt of colonoscopy for a positive FIT result; screening cessation (at age $70 \mathrm{y}$ ); migration; and death. Colonoscopies performed within 1 year of a positive FIT result and surgical treatments performed within 1 year of a positive colonoscopy were considered part of a single screening episode. The last date of follow-up evaluation of positive FIT results was December 31, 2018. 
Table 1. Total Screening Population: Number of Subjects Invited to and Participating in Five Organizational Screening Rounds, the Number of Subjects With Positive FIT Results, of Subjects Undergoing Colonoscopic Assessment, of Subjects With Successful Cecal Intubation, and of Subjects Diagnosed With Colorectal Adenoma and Colorectal Cancer, by Sex and Screening Round

\begin{tabular}{|c|c|c|c|c|c|c|c|c|c|c|}
\hline & \multicolumn{5}{|c|}{ Males } & \multicolumn{5}{|c|}{ Females } \\
\hline & $1 \mathrm{st}$ & 2nd & 3rd & 4th & 5 th & $1 \mathrm{st}$ & 2nd & $3 r d$ & 4th & 5th \\
\hline Invited & 405,639 & 477,656 & 489,301 & 521,819 & 525,698 & 439,466 & 512,661 & 529,538 & 562,309 & 565,873 \\
\hline Participating & 183,503 & 229,254 & 244,577 & 258,001 & 261,179 & 214,941 & 266,779 & 283,270 & 299,020 & 302,663 \\
\hline With positive FIT results & 13,108 & 14,397 & 12,170 & 12,140 & 13,370 & 9974 & 11,491 & 10,176 & 10,505 & 11,893 \\
\hline With colonoscopic assessment & 10,397 & 11,496 & 9967 & 9840 & 10,624 & 7586 & 8792 & 8188 & 8379 & 9301 \\
\hline $\begin{array}{l}\text { With successful cecal intubation } \\
\text { With adenoma }\end{array}$ & 9853 & 11,053 & 9445 & 9487 & 10,289 & 7015 & 8382 & 7732 & 8100 & 8969 \\
\hline Advanced adenoma & 4062 & 3729 & 3218 & 3063 & 2930 & 2160 & 2046 & 1843 & 1786 & 1754 \\
\hline Total adenoma & 5668 & 5907 & 5014 & 4710 & 4784 & 3082 & 3296 & 3000 & 2835 & 2899 \\
\hline \multicolumn{11}{|l|}{ With colorectal cancer ${ }^{a}$} \\
\hline Stage I & 383 & 313 & 198 & 152 & 139 & 252 & 188 & 126 & 115 & 109 \\
\hline Stage II & 136 & 90 & 81 & 59 & 48 & 73 & 66 & 62 & 46 & 47 \\
\hline Stage III & 145 & 119 & 66 & 72 & 53 & 98 & 88 & 69 & 72 & 51 \\
\hline Stage IV ${ }^{a}$ & NA & 5 & 9 & 12 & 11 & NA & 2 & 8 & 7 & 4 \\
\hline Stage unknown & 191 & 69 & 55 & 41 & 59 & 89 & 35 & 36 & 27 & 28 \\
\hline Total colorectal cancer & 855 & 596 & 409 & 336 & 310 & 512 & 379 & 301 & 267 & 239 \\
\hline With advanced neoplasia ${ }^{b}$ & 4917 & 4325 & 3627 & 3399 & 3240 & 2672 & 2425 & 2144 & 2053 & 1993 \\
\hline
\end{tabular}

NOTE. All data are from the Emilia-Romagna Region colorectal cancer screening program (2005-2014).

FIT, fecal immunochemical test; NA, not available.

${ }^{a}$ During the first screening round, data for stage IV colorectal cancer in the total screening population were not collected.

${ }^{b}$ Advanced neoplasia indicates advanced adenoma and colorectal cancer.

With respect to the total screening population, the performance measures of screening were calculated in five 2-year screening rounds (organizational rounds). The population was treated as an open cohort.

\section{Data Sources}

With respect to the total screening population, study data were taken from the annual national surveys conducted by the National Centre for Screening Monitoring, which collects standard data from local screening units. Data on the detection of CRC by disease site were not collected.

Data for compliant participants were obtained from the Information System for the Surveillance of Colorectal Screening (flusso informativo Screening Colon-Retto) of the Department of Health of the Regional Administration. Every 4 months, each health care district screening unit provides electronic records for all subjects in the target population. The System is an anonymous relational database created by record-linking multiple data sets (population list, invitations, FITs, colonoscopies, diagnoses, surgical treatments, and vital status). The findings of each screening episode are classified as follows: CRC, advanced adenoma ( $\geq 1 \mathrm{~cm}$ in diameter, or villous/ tubulovillous type, or with high-grade dysplasia), nonadvanced adenoma, or negative. In the case of CRC, tumor stage and disease site were recorded. Tumor stage was classified according to the American Joint Committee on Cancer TNM staging system, sixth edition. Disease site was classified as the proximal colon (from the cecum to the transverse colon), distal colon (from the splenic flexure to the sigmoid colon), and rectum (rectosigmoid junction and rectum).$^{10}$ Because a successful cecal intubation was achieved in a proportion of patients close to $100 \%$, this information was not included in the download file.

\section{Performance Measures}

The primary study end points included the following performance measures: (1) the proportion of positive FIT results (ie, the percentage of subjects with a completed FIT who had a positive result); (2) the DR of advanced adenoma, CRC, and advanced neoplasia (including CRC and advanced adenoma) (ie, the proportion of subjects with a completed FIT who were diagnosed with these lesions per 1000 screenees); and (3) the PPV for adenoma (also referred to as the adenoma detection rate), ${ }^{11}$ advanced adenoma, CRC, and advanced neoplasia at colonoscopy (ie, the percentage of subjects undergoing colonoscopy who were diagnosed with nonadvanced/advanced adenoma, advanced adenoma, CRC, and advanced neoplasia).

\section{Statistical Analysis}

Analysis was truncated at the fifth screening round because the length of follow-up evaluation available in the case of positive FIT results in the sixth round was insufficient for more than $50 \%$ of subjects.
291 
Table 2. Total Screening Population: Participation Rate, Proportion of Positive FIT Results, Colonoscopic Assessment Rate, Successful Cecal Intubation Rate, DR of Colorectal Adenoma and Colorectal Cancer, and PPV for Colorectal Adenoma and Colorectal Cancer, by Sex and Screening Round

\begin{tabular}{|c|c|c|c|c|c|c|c|c|c|c|}
\hline & \multicolumn{5}{|c|}{ Males } & \multicolumn{5}{|c|}{ Females } \\
\hline & 1st & 2nd & 3rd & 4th & 5 th & $1 \mathrm{st}$ & 2nd & 3rd & 4th & 5th \\
\hline Participation rate, \% & 45.2 & 48.0 & 50.0 & 49.4 & 49.7 & 48.9 & 52.0 & 53.5 & 53.2 & 53.5 \\
\hline Proportion of positive FIT results, $\%$ & 7.1 & 6.3 & 5.0 & 4.7 & 5.1 & 4.6 & 4.3 & 3.6 & 3.5 & 3.9 \\
\hline Colonoscopic assessment rate, $\%$ & 79.3 & 79.8 & 81.9 & 81.1 & 79.5 & 76.1 & 76.5 & 80.5 & 79.8 & 78.2 \\
\hline Successful cecal intubation rate, \% & 94.8 & 96.1 & 94.8 & 96.4 & 96.8 & 92.5 & 95.3 & 94.4 & 96.7 & 96.4 \\
\hline DR of advanced adenoma (per 1000) & 22.1 & 16.3 & 13.2 & 11.9 & 11.2 & 10.0 & 7.7 & 6.5 & 6.0 & 5.8 \\
\hline DR of colorectal cancer (per 1000) & 4.7 & 2.6 & 1.7 & 1.3 & 1.2 & 2.4 & 1.4 & 1.1 & 0.9 & 0.8 \\
\hline DR of advanced neoplasia ${ }^{a}$ (per 1000) & 26.8 & 18.9 & 14.8 & 13.2 & 12.4 & 12.4 & 9.1 & 7.6 & 6.9 & 6.6 \\
\hline PPV for adenoma, ${ }^{b} \%$ & 54.5 & 51.4 & 50.3 & 47.9 & 45.0 & 40.6 & 37.5 & 36.6 & 33.8 & 31.2 \\
\hline PPV for advanced adenoma, $\%$ & 39.1 & 32.4 & 32.3 & 31.1 & 27.6 & 28.5 & 23.3 & 22.5 & 21.3 & 18.9 \\
\hline PPV for colorectal cancer, $\%$ & 8.2 & 5.2 & 4.1 & 3.4 & 2.9 & 6.7 & 4.3 & 3.7 & 3.2 & 2.6 \\
\hline PPV for advanced neoplasia, \% & 47.3 & 37.6 & 36.4 & 34.5 & 30.5 & 35.2 & 27.6 & 26.2 & 24.5 & 21.4 \\
\hline DR of stage I colorectal cancer (per 1000) & 2.1 & 1.4 & 0.8 & 0.6 & 0.5 & 1.2 & 0.7 & 0.4 & 0.4 & 0.4 \\
\hline DR of stage II colorectal cancer (per 1000) & 0.7 & 0.4 & 0.3 & 0.2 & 0.2 & 0.3 & 0.2 & 0.2 & 0.2 & 0.2 \\
\hline DR of stage III colorectal cancer (per 1000) & 0.8 & 0.5 & 0.3 & 0.3 & 0.2 & 0.5 & 0.3 & 0.2 & 0.2 & 0.2 \\
\hline DR of stage IV colorectal cancer (per 1000) ${ }^{C}$ & NA & $<0.1$ & $<0.1$ & $<0.1$ & $<0.1$ & NA & $<0.1$ & $<0.1$ & $<0.1$ & $<0.1$ \\
\hline
\end{tabular}

NOTE. All data are from the Emilia-Romagna Region colorectal cancer screening program (2005-2014). The participation rate is per 100 subjects invited. The proportion of positive FIT results is per 100 subjects undergoing FIT screening. The colonoscopic assessment rate is per 100 subjects with positive FIT results. The successful cecal intubation rate is per 100 subjects with positive FIT results undergoing colonoscopic assessment. The DRs of advanced adenoma, colorectal cancer, and advanced neoplasia are per 1000 subjects undergoing FIT screening. The PPVs are per 100 subjects with positive FIT results undergoing colonoscopic assessment. Anal cancer cases were excluded from the DR of colorectal cancer by tumor stage.

DR, detection rate; FIT, fecal immunochemical test; NA, not available; PPV, positive predictive value.

${ }^{a}$ Advanced neoplasia indicates advanced adenoma and colorectal cancer.

${ }^{b}$ Also referred to as the adenoma detection rate.

${ }^{c}$ During the first screening round, data for stage IV colorectal cancer in the total screening population were not collected.

Multivariate Poisson regression models were built to estimate the rate ratio, with $95 \%$ CIs, for the association between the screening round and each of the earlierdescribed performance measures. The second round was used as a reference category.

Further analyses were restricted to the DR of CRC. Multinomial logistic models were built to estimate the probability of detection of CRC by disease site and tumor stage compared with no detection. The probability was expressed as a relative risk ratio, with $95 \%$ CIs, using the second screening round as a reference category. Anal cancer cases were excluded from the estimate of the probability of detection of CRC by disease site and tumor stage. All models were adjusted for age.

All analyses were performed using SAS Enterprise Guide (version 5.1; SAS Institute, Inc, Cary, NC) and STATA (version 15.1, Stata Corporation, College Station, TX).

This study received Institutional Review Board approval from the Romagna Cancer Institute (protocol ID, L1P2043).

\section{Results}

\section{Total Screening Population}

Table 1 shows the absolute numbers of all subjects who were invited to screening, who participated, who tested positive, and who were diagnosed with adenoma and CRC in 5 organizational screening rounds. The resulting performance measures are shown in Table 2 .

\section{Compliant Participants: Numbers and Rates}

Tables 3 to 6 consider only compliant participants. Table 3 provides their absolute numbers as well as the numbers of subjects with positive FIT results and with screen-detected disease.

Table 4 shows the performance measures under study, namely, the participation rate, the proportion of positive FIT results, the colonoscopic assessment rate, the DR of (and the PPV for) adenoma, advanced adenoma, CRC, and advanced neoplasia, and the DR of CRC by disease site and tumor stage. Contrasting these data with those from the total screening population, the second and subsequent rounds among compliant participants of both sexes were characterized by a smaller proportion of positive FIT results and a generally lower DR of advanced adenoma. The PPVs for advanced adenoma and advanced neoplasia also were lower.

\section{Compliant Participants: Trends Across Rounds}

Table 5 shows the rate ratios for the association between the screening round and the main of the earlier- Q17 described measures. The expected decrease between 
Table 3. Compliant Participants: Number of Subjects Eligible to, Invited to, and Participating in Five Screening Rounds, Number of Subjects With Positive FIT Results, Undergoing Colonoscopic Assessment, With Successful Cecal Intubation, and Diagnosed With Colorectal Adenoma and Colorectal Cancer, by Sex and Screening Round

\begin{tabular}{|c|c|c|c|c|c|c|c|c|c|c|}
\hline & \multicolumn{5}{|c|}{ Males } & \multicolumn{5}{|c|}{ Females } \\
\hline & 1st & 2nd & $3 r d$ & 4th & 5 th & 1st & 2nd & $3 r d$ & 4th & 5th \\
\hline Eligible & 501,826 & 215,681 & 129,396 & 97,675 & 72,054 & 535,706 & 254,292 & 154,635 & 118,134 & 88,491 \\
\hline Invited & 474,319 & 176,420 & 110,717 & 82,133 & 60,260 & 508,912 & 208,163 & 133,226 & 100,116 & 74,713 \\
\hline Participants & 229,742 & 135,508 & 101,506 & 76,941 & 57,041 & 264,445 & 159,644 & 121,662 & 93,454 & 70,437 \\
\hline With positive FIT results & 16,434 & 7024 & 4339 & 3375 & 2686 & 12,244 & 5909 & 4017 & 3198 & 2695 \\
\hline With colonoscopic assessment & 14,061 & 6112 & 3831 & 3039 & 2353 & 10,153 & 5009 & 3528 & 2820 & 2340 \\
\hline With successful cecal intubation & NA & NA & NA & NA & NA & NA & NA & NA & NA & NA \\
\hline \multicolumn{11}{|l|}{ With adenoma } \\
\hline Advanced adenoma & 5726 & 1819 & 1034 & 848 & 592 & 3099 & 1024 & 651 & 509 & 402 \\
\hline Total adenoma & 7912 & 3022 & 1779 & 1451 & 1078 & 4350 & 1731 & 1174 & 941 & 770 \\
\hline \multicolumn{11}{|l|}{ With colorectal cancer } \\
\hline Proximal colon & 222 & 70 & 58 & 42 & 37 & 152 & 71 & 68 & 37 & 44 \\
\hline Distal colon & 600 & 99 & 47 & 24 & 22 & 362 & 53 & 40 & 24 & 13 \\
\hline Rectum & 229 & 62 & 39 & 38 & 23 & 110 & 44 & 26 & 16 & 15 \\
\hline Anus $^{a}$ & 2 & 1 & 0 & 1 & 2 & 2 & 2 & 0 & 0 & 0 \\
\hline Site unknown & 1 & 0 & 0 & 0 & 0 & 2 & 0 & 0 & 0 & 0 \\
\hline Stage I & 572 & 114 & 78 & 54 & 42 & 346 & 79 & 54 & 38 & 36 \\
\hline Stage II & 196 & 47 & 35 & 17 & 17 & 106 & 27 & 26 & 16 & 10 \\
\hline Stage III & 184 & 46 & 21 & 22 & 13 & 133 & 55 & 39 & 21 & 17 \\
\hline Stage IV & 42 & 7 & 2 & 2 & 3 & 13 & 2 & 6 & 0 & 1 \\
\hline Stage unknown & 58 & 17 & 8 & 9 & 7 & 28 & 5 & 9 & 2 & 8 \\
\hline Total colorectal cancer & 1054 & 232 & 144 & 105 & 84 & 628 & 170 & 134 & 77 & 72 \\
\hline With advanced neoplasia ${ }^{b}$ & 6780 & 2051 & 1178 & 953 & 676 & 3727 & 1194 & 785 & 586 & 474 \\
\hline
\end{tabular}

NOTE. All data are from the Emilia-Romagna Region colorectal cancer screening program (2005-2016). A compliant participant was defined as one who had 2, 3 , 4 , or 5 consecutive FITs at standard intervals (ie, $2 \mathrm{y} \pm 6 \mathrm{mo}$ ). A total of 1848 males and 2143 females with negative FIT results in the fourth screening round were considered not eligible for the fifth round because the length of follow-up time available in the case of positive FIT results was insufficient. The discrepancy between the number eligible and the number invited in each round is accounted for by subjects who died or migrated before invitation, subjects who were invited or re-invited at age 70 or older, and subjects who were re-invited more than 2.5 years after the previous negative FIT.

FIT, fecal immunochemical test; NA, not available.

${ }^{a}$ Anal cancer cases were excluded from the number of cases by tumor stage.

${ }^{b}$ Advanced neoplasia indicates advanced adenoma and colorectal cancer.

the first and the second round was confirmed for all of these, with the exception of the colonoscopic assessment rate. Between the second and the third round, a further $20 \%$ to $30 \%$ decrease was observed for males in the proportion of positive FIT results and in all DRs. PPVs decreased by $10 \%$ or less. Among females, these measures decreased to a lesser extent.

At the fourth and fifth round, the DRs and the PPV for adenoma stabilized. Conversely, a further, albeit limited, decrease was observed for both sexes in the PPV for advanced adenoma, colorectal cancer, and advanced neoplasia. The tests for interaction between sex and screening round confirmed that the downward trend in most measures was more pronounced for males.

In Table 6, the relative risk ratios for the association between the screening round and the DR of CRC by disease site and tumor stage are shown. For both sexes, the DR of proximal colon cancer did not change further after the second round, whereas the yield of distal disease continued to decrease until the fourth (males) and fifth (females) rounds. This observation should be related to the fact that the DR of proximal colon cancer, after plateauing, remained at a higher absolute level than that of distal colon cancer, especially among females
(Table 4). Regarding tumor stage, the DR of stage I and stage II CRC stabilized, for both sexes, only at the fourth round.

\section{Discussion}

Compared with the total screening population, compliant participants in the second and subsequent screening rounds had a lower proportion of positive FIT results and generally lower values for the DR of advanced adenoma and the PPV for advanced adenoma and advanced neoplasia. Data for organizational screening rounds refer to total participants, including subjects at their first FIT and subjects with occasional participation who had a screening interval longer than the standard. The prevalence of preclinical disease was higher in never-screened subjects and, albeit lower, increased with increasing screening intervals among ever-screened subjects. Thus, the earlier-described findings were expected.

With respect to compliant participants, the length of follow-up evaluation and the epidemiologic background of this study are comparable with the 2 complementary 
Table 4. Compliant Participants: Participation Rate, Proportion of Positive FIT Results, Colonoscopic Assessment Rate, Successful Cecal Intubation Rate, DR of Colorectal Adenoma and Colorectal Cancer, and PPV for Colorectal Adenoma and Colorectal Cancer, by Sex and Screening Round

\begin{tabular}{|c|c|c|c|c|c|}
\hline & \multicolumn{5}{|c|}{ Round } \\
\hline & $1 \mathrm{st}$ & 2nd & 3rd & 4th & 5th \\
\hline \multicolumn{6}{|l|}{ Males } \\
\hline Participation rate, $\%$ & 48.4 & 76.8 & 91.7 & 93.7 & 94.7 \\
\hline Proportion of positive FIT results, $\%$ & 7.2 & 5.2 & 4.3 & 4.4 & 4.7 \\
\hline Colonoscopic assessment rate, $\%$ & 85.6 & 87.0 & 88.3 & 90.0 & 87.6 \\
\hline Successful cecal intubation rate, $\%$ & NA & NA & NA & NA & NA \\
\hline DR of advanced adenoma (per 1000) & 24.9 & 13.4 & 10.2 & 11.0 & 10.4 \\
\hline DR of colorectal cancer (per 1000) & 4.6 & 1.7 & 1.4 & 1.4 & 1.5 \\
\hline DR of advanced neoplasia ${ }^{a}$ (per 1000) & 29.5 & 15.1 & 11.6 & 12.4 & 11.9 \\
\hline PPV for adenoma, ${ }^{b} \%$ & 56.3 & 49.4 & 46.4 & 47.7 & 45.8 \\
\hline PPV for advanced adenoma, $\%$ & 40.7 & 29.8 & 27.0 & 27.9 & 25.2 \\
\hline PPV for colorectal cancer, $\%$ & 7.5 & 3.8 & 3.8 & 3.5 & 3.6 \\
\hline PPV for advanced neoplasia, ${ }^{a} \%$ & 48.2 & 33.6 & 30.7 & 31.4 & 28.7 \\
\hline DR of proximal colon cancer (per 1000) & 1.0 & 0.5 & 0.6 & 0.5 & 0.6 \\
\hline DR of distal colon cancer (per 1000) & 2.6 & 0.7 & 0.5 & 0.3 & 0.4 \\
\hline DR of rectal cancer (per 1000) & 1.0 & 0.5 & 0.4 & 0.5 & 0.4 \\
\hline DR of stage I colorectal cancer (per 1000) & 2.5 & 0.8 & 0.8 & 0.7 & 0.7 \\
\hline DR of stage II colorectal cancer (per 1000) & 0.9 & 0.3 & 0.3 & 0.2 & 0.3 \\
\hline DR of stage III colorectal cancer (per 1000) & 0.8 & 0.3 & 0.2 & 0.3 & 0.2 \\
\hline DR of stage IV colorectal cancer (per 1000) & 0.2 & 0.1 & $<0.1$ & $<0.1$ & 0.1 \\
\hline \multicolumn{6}{|l|}{ Females } \\
\hline Participation rate, \% & 52.0 & 76.7 & 91.3 & 93.3 & 94.3 \\
\hline Proportion of positive FIT results, $\%$ & 4.6 & 3.7 & 3.3 & 3.4 & 3.8 \\
\hline Colonoscopic assessment rate, $\%$ & 82.9 & 84.8 & 87.8 & 88.2 & 86.8 \\
\hline Successful cecal intubation rate, $\%$ & NA & NA & NA & NA & NA \\
\hline DR of advanced adenoma (per 1000) & 11.7 & 6.4 & 5.4 & 5.5 & 5.7 \\
\hline DR of colorectal cancer (per 1000) & 2.4 & 1.1 & 1.1 & 0.8 & 1.0 \\
\hline DR of advanced neoplasia ${ }^{a}$ (per 1000) & 14.1 & 7.5 & 6.5 & 6.3 & 6.7 \\
\hline PPV for adenoma, ${ }^{b} \%$ & 42.8 & 34.6 & 33.3 & 33.4 & 32.9 \\
\hline PPV for advanced adenoma, $\%$ & 30.5 & 20.4 & 18.5 & 18.0 & 17.2 \\
\hline PPV for colorectal cancer, $\%$ & 6.2 & 3.4 & 3.8 & 2.7 & 3.1 \\
\hline PPV for advanced neoplasia, ${ }^{a} \%$ & 36.7 & 23.8 & 22.3 & 20.8 & 20.3 \\
\hline DR of proximal colon cancer (per 1000) & 0.6 & 0.4 & 0.6 & 0.4 & 0.6 \\
\hline DR of distal colon cancer (per 1000) & 1.4 & 0.3 & 0.3 & 0.3 & 0.2 \\
\hline DR of rectal cancer (per 1000) & 0.4 & 0.3 & 0.2 & 0.2 & 0.2 \\
\hline DR of stage I colorectal cancer (per 1000) & 1.3 & 0.5 & 0.4 & 0.4 & 0.5 \\
\hline DR of stage II colorectal cancer (per 1000) & 0.4 & 0.2 & 0.2 & 0.2 & 0.1 \\
\hline DR of stage III colorectal cancer (per 1000) & 0.5 & 0.3 & 0.3 & 0.2 & 0.2 \\
\hline DR of stage IV colorectal cancer (per 1000) & $<0.1$ & $<0.1$ & $<0.1$ & 0 & $<0.1$ \\
\hline
\end{tabular}

NOTE. All data are from the Emilia-Romagna Region colorectal cancer screening program (2005-2016). A compliant participant was defined as one who had 2, 3 , 4 , or 5 consecutive FITs at standard intervals (ie, $2 \mathrm{y} \pm 6 \mathrm{mo}$ ). The participation rate is per 100 subjects invited. The proportion of positive FIT results is per 100 subjects undergoing FIT screening. The colonoscopic assessment rate is per 100 subjects with positive FIT results. The successful cecal intubation rate, if available, would be per 100 subjects with positive FIT results undergoing colonoscopic assessment. The DRs of advanced adenoma, colorectal cancer, and advanced neoplasia are per 1000 subjects undergoing FIT screening. The PPVs are per 100 subjects with positive FIT results undergoing colonoscopic assessment. Anal cancer cases were excluded from the number of cases by tumor stage.

DR, detection rate; FIT, fecal immunochemical test; NA, not available; PPV, positive predictive value.

${ }^{a}$ Advanced neoplasia indicates advanced adenoma and colorectal cancer.

${ }^{b}$ Also referred to as the adenoma detection rate.

studies by Zorzi et al, ${ }^{6,7}$ although they pooled males and females in their study. In their data, the proportion of positive FIT results, the DR of advanced adenoma, and the PPV for advanced neoplasia decreased between the first and the second rounds and then stabilized, whereas the DR of CRC decreased until the third round before plateauing. ${ }^{6}$ Our results followed a similar pattern, but all DRs as well as the proportion of positive FIT results continued to decrease until the third round. In addition, this trend was more pronounced for males.
Regarding the trends in the DR by disease site, our findings were practically identical to those reported by Zorzi et $\mathrm{al}^{7}$ for both sexes combined. The detection of proximal colon cancer decreased between the first and the second rounds and then stabilized, whereas the detection of distal colon cancer decreased over 5 rounds. In addition, the DR of proximal colon cancer remained at a higher level than that of distal colon cancer, especially among females. Many colonoscopy-verified diagnostic studies $^{12}$ and studies on the proportional incidence of 
Table 6. Compliant Participants: Comparison of the First, Third, Fourth, and Fifth Screening Rounds Vs the Second Round for the DR of Colorectal Cancer by Disease Site, Tumor Stage, and Sex

\begin{tabular}{|c|c|c|c|c|c|}
\hline & \multicolumn{5}{|c|}{ Round } \\
\hline & $1 \mathrm{st}$ & 2nd & 3rd & 4th & 5 th \\
\hline \multicolumn{6}{|l|}{ Males } \\
\hline DR of proximal colon cancer & $2.01(1.54-2.63)$ & 1.00 (ref) & $1.03(0.73-1.46)$ & $0.91(0.62-1.34)$ & $1.00(0.67-1.50)$ \\
\hline DR of distal colon cancer & $3.80(3.07-4.70)$ & 1.00 (ref) & $0.60(0.42-0.84)$ & $0.37(0.24-0.58)$ & $0.43(0.27-0.69)$ \\
\hline DR of rectal cancer & $2.32(1.75-3.07)$ & 1.00 (ref) & $0.79(0.53-1.18)$ & $0.96(0.64-1.44)$ & $0.74(0.46-1.20)$ \\
\hline DR of stage I colorectal cancer & $3.15(2.57-3.85)$ & 1.00 (ref) & $0.86(0.64-1.15)$ & $0.73(0.53-1.02)$ & $0.72(0.51-1.03)$ \\
\hline DR of stage II colorectal cancer & $2.66(1.93-3.65)$ & 1.00 (ref) & $0.92(0.59-1.43)$ & $0.54(0.31-0.94)$ & $0.68(0.39-1.18)$ \\
\hline DR of stage III colorectal cancer & $2.51(1.81-3.46)$ & 1.00 (ref) & $0.57(0.34-0.96)$ & $0.74(0.44-1.23)$ & $0.55(0.30-1.02)$ \\
\hline DR of stage IV colorectal cancer & $3.73(1.68-8.29)$ & 1.00 (ref) & $0.36(0.07-1.74)$ & $0.45(0.09-2.19)$ & $0.88(0.22-3.45)$ \\
\hline \multicolumn{6}{|l|}{ Females } \\
\hline DR of proximal colon cancer & $1.39(1.05-1.85)$ & 1.00 (ref) & $1.16(0.83-1.61)$ & $0.76(0.51-1.13)$ & $1.11(0.76-1.62)$ \\
\hline DR of distal colon cancer & $4.37(3.27-5.84)$ & 1.00 (ref) & $0.93(0.62-1.40)$ & $0.68(0.42-1.10)$ & $0.46(0.25-0.85)$ \\
\hline DR of rectal cancer & $1.57(1.10-2.24)$ & 1.00 (ref) & $0.74(0.46-1.20)$ & $0.56(0.32-0.99)$ & $0.66(0.37-1.18)$ \\
\hline DR of stage I colorectal cancer & $2.81(2.20-3.59)$ & 1.00 (ref) & $0.84(0.59-1.18)$ & $0.71(0.48-1.05)$ & $0.83(0.56-1.23)$ \\
\hline DR of stage II colorectal cancer & $2.53(1.66-3.86)$ & 1.00 (ref) & $1.17(0.68-2.01)$ & $0.88(0.47-1.63)$ & $0.68(0.33-1.42)$ \\
\hline DR of stage III colorectal cancer & $1.54(1.12-2.12)$ & 1.00 (ref) & $0.88(0.58-1.32)$ & $0.58(0.35-0.96)$ & $0.60(0.35-1.03)$ \\
\hline DR of stage IV colorectal cancer & $4.15(0.86-19.99)$ & 1.00 (ref) & $3.70(0.80-17.12)$ & 0 & $0.90(0.09-9.36)$ \\
\hline
\end{tabular}

NOTE. All data are from the Emilia-Romagna Region colorectal cancer screening program (2005-2016). A compliant participant was defined as one who had 2, 3 , 4 , or 5 consecutive FITs at standard intervals (ie, $2 \mathrm{y} \pm 6 \mathrm{mo}$ ). Numbers are as relative risk ratios from multinomial logistic regression models. Numbers in parentheses are $95 \%$ Cls. Anal cancer cases were excluded from the number of cases by tumor stage. $\mathrm{DR}$, detection rate.

patients. ${ }^{20}$ Our results suggest that compliant long-term participation in FIT screening must be included among the latter.

Fourth, these findings have relevance to the hypothesis of using risk prediction models based on established risk factors for allocation to personalized screening intervals and/or protocols. A systematic review showed that screening history is not among the most commonly used risk factors (age, sex, family history, obesity, and smoking). ${ }^{21}$ For subjects allocated to standard FIT screening, however, a compliant participation in 5 to 6 rounds may alter the risk of disease to a substantial extent. We agree with the view that screening history cannot be ignored. ${ }^{22}$ The number of previous negative tests, if combined with the amount of hemoglobin in negative FIT, could identify a small group with very high risk and a large population with minimal risk. ${ }^{23}$ For example, Zorzi et $\mathrm{al}^{6}$ proposed increasing the cut-off value for positivity or extending the screening interval for women and younger individuals after 3 negative FITs (provided that the incidence of interval cancer does not exceed the maximum acceptable levels).

This study had some major weaknesses. First, the sharp decrease in the number of subjects undergoing FIT between the first and the fifth round-inherent to the study design-caused an increasing random variation in results. The decrease in the number of compliant participants, however, mainly was owing to external factors (ie, late invitation, screening cessation at age 70 years, migration, and death). These factors accounted, for example, for more than $75 \%$ of subjects who participated in the fourth round, but not in the fifth round (data not shown). This suggests that the findings of the last rounds were not affected by major selection biases.

Second, previous studies have shown that the performance measures of follow-up rounds depend on the number of FITs and the chosen cut-off values. With lower cut-off values or with 2 FIT screenings, more advanced neoplasias were detected at the baseline round, and fewer during subsequent rounds. ${ }^{8}$ Consequently, our results cannot be generalized to different screening protocols.

Theoretically, studies of this type may be affected by another potential bias that relates to those subjects with positive FIT results and negative colonoscopy who are re-invited to screening some rounds later. These subjects, who are at low risk of disease, might influence the results at subsequent rounds. It must be noted, however, that this cannot be the case for the present study, in which follow-up evaluation ceased on the day of receipt of colonoscopy for a positive FIT result.

In summary, compliant participants had a lower Q20 proportion of positive FIT results than the total screening population. For them, some major performance measures continued to decrease even after the expected decrease between the first and the second rounds. Between the second and the third rounds, the proportion of positive FIT results and the DR of advanced adenoma, CRC, and advanced neoplasia decreased by $20 \%$ to $30 \%$ among males. For both sexes, the DR of distal colon cancer decreased until the fourth (males) and fifth rounds (females). These findings add circumstantial evidence that FIT screening prevents the progression of an appreciable subset of advanced 
adenomas, and have implications for the communication of benefits and harms of the screening process, the planning of endoscopy services, and the development of risk-based screening strategies.

\section{References}

1. Crotta S, Segnan N, Paganin S, et al. High rate of advanced adenoma detection in 4 rounds of colorectal cancer screening with the fecal immunochemical test. Clin Gastroenterol Hepatol 2012;10:633-638.

2. Parente F, Boemo C, Ardizzoia A, et al. Outcomes and cost evaluation of the first two rounds of a colorectal cancer screening program based on immunochemical fecal occult blood test in northern Italy. Endoscopy 2013;45:27-34.

3. Kapidzic A, Grobbee EJ, Hol L, et al. Attendance and yield over three rounds of population-based fecal immunochemical test screening. Am J Gastroenterol 2014;109:1257-1264.

4. Stegeman I, van Doorn SC, Mundt MW, et al. Participation, yield, and interval carcinomas in three rounds of biennial FIT-based colorectal cancer screening. Cancer Epidemiol 2015;39:388-393.

5. Kapidzic A, van Roon AH, van Leerdam ME, et al. Attendance and diagnostic yield of repeated two-sample faecal immunochemical test screening for colorectal cancer. Gut 2017; 66:118-123.

6. Zorzi M, Hassan C, Capodaglio G, et al. Long-term performance of colorectal cancer screening programmes based on the faecal immunochemical test. Gut 2018;67:2124-2130.

7. Zorzi M, Hassan C, Capodaglio G, et al. Divergent long-term detection rates of proximal and distal advanced neoplasia in fecal immunochemical test screening programs: a retrospective cohort study. Ann Intern Med 2018;169:602-609.

8. Schreuders EH, Grobbee EJ, Nieuwenburg SAV, et al. Multiple rounds of one sample versus two sample faecal immunochemical test-based colorectal cancer screening: a populationbased study. Lancet Gastroenterol Hepatol 2019;4:622-631.

9. Mancini S, Ravaioli A, Falcini F, et al. Strategies for delivery of faecal occult blood test kits and participation to colorectal cancer screening in the Emilia-Romagna Region of Italy. Eur $\mathrm{J}$ Cancer Care (Engl) 2018;27.

10. Zorzi M, Mangone L, Sassatelli R, et al. Incidence trends of colorectal cancer in the early 2000s in Italy. Figures from the IMPATTO study on colorectal cancer screening. Epidemiol Prev 2015;39(Suppl 1):115-125.

11. Wieszczy P, Regula J, Kaminski MF. Adenoma detection rate and risk of colorectal cancer. Best Pract Res Clin Gastroenterol 2017;31:441-446.

12. Hirai HW, Tsoi KK, Chan JY, et al. Systematic review with metaanalysis: faecal occult blood tests show lower colorectal cancer detection rates in the proximal colon in colonoscopy-verified diagnostic studies. Aliment Pharmacol Ther 2016;43:755-764.

13. Zappa M, Castiglione G, Paci E, et al. Measuring interval cancers in population-based screening using different assays of fecal occult blood testing: the District of Florence experience. Int J Cancer 2001;92:151-154.

14. Zorzi M, Fedato C, Grazzini G, et al. High sensitivity of five colorectal screening programmes with faecal immunochemical test in the Veneto Region. Italy. Gut 2011;60:944-949.

15. Morikawa $\mathrm{T}$, Kato $\mathrm{J}$, Yamaji $\mathrm{Y}$, et al. A comparison of the immunochemical fecal occult blood test and total colonoscopy in the asymptomatic population. Gastroenterology 2005; 129:422-428.
16. Haug $U$, Kuntz KM, Knudsen $A B$, et al. Sensitivity of immunochemical faecal occult blood testing for detecting leftvs right-sided colorectal neoplasia. $\mathrm{Br} J$ Cancer 2011; 104:1779-1785.

17. Giorgi Rossi $P$, Vicentini $M$, Sacchettini $C$, et al. Impact of screening program on incidence of colorectal cancer: a cohort study in Italy. Am J Gastroenterol 2015;110:1359-1366.

18. Ventura L, Mantellini $P$, Grazzini G, et al. The impact of immunochemical faecal occult blood testing on colorectal cancer incidence. Dig Liver Dis 2014;46:82-86.

19. Al-Najami I, Rancinger CP, Larsen MK, et al. The diagnostic yield of colonoscopy stratified by indications. Gastroenterol Res Pract 2017;2017:4910143.

20. Parente F, Marino B, llardo A, et al. A combination of faecal tests for the detection of colon cancer: a new strategy for an appropriate selection of referrals to colonoscopy? A prospective multicentre Italian study. Eur J Gastroenterol Hepatol 2012; 24:1145-1152.

21. Ma GK, Ladabaum U. Personalizing colorectal cancer screening: a systematic review of models to predict risk of colorectal neoplasia. Clin Gastroenterol Hepatol 2014; 12:1624-1634.

22. Cooper JA, Moss SM, Smith S, et al. FIT for the future: a case for risk-based colorectal cancer screening using the faecal immunochemical test. Colorectal Dis 2016;18:650-653.

23. Senore C, Zappa M, Campari C, et al. Faecal haemoglobin concentration among subjects with negative FIT results is associated with the detection rate of neoplasia at subsequent rounds: a prospective study in the context of population based screening programmes in Italy. Gut 2020;69:523-530.

\section{Reprint requests}

Address requests for reprints to: Lauro Bucchi, MD, Romagna Cancer Registry, Romagna Cancer Institute, Istituto Scientifico Romagnolo per lo Studio e la Cura dei Tumori, IRCCS, Meldola, Forlì, Italy. e-mail: lauro.bucchii@irst.emr.it; fax: (39) 543739459

\section{Acknowledgments}

CRediT Authorship Contributions: The membership of the Emilia-Romagna Q7 Region Workgroup for Colorectal Screening Evaluation is as follows: Stefano Q6 Ferretti (University of Ferrara and Local Health Authority, Ferrara, Italy); Francesca Mezzetti, Enza Di Felice, and Priscilla Sassoli de Bianchi (Department of Health, Regional Administration, Emilia-Romagna Region, Bologna, Italy); Chiara Ventura (ICT Service, Technologies, and Healthcare Facilities, Depart- Q8 ment of Health, Regional Administration, Emilia-Romagna Region, Bologna, Italy), Flavia Baldacchini, Lauro Bucchi, Americo Colamartini, Orietta Giuliani, Silvia Mancini, Alessandra Ravaioli, and Rosa Vattiato (Romagna Cancer Registry, Romagna Cancer Institute, IRST, IRCCS, Meldola, Forli, Italy); Fabio Falcini (Romagna Cancer Registry, Romagna Cancer Institute, IRST, IRCCS, Meldola, Forli, Italy, and Local Health Authority, Forlì, Italy), Dario Signorelli, Giovanni Aragona, Francesca Pennini, Giorgio Chiaranda, and Pietro Seghin (Local Health Authority, Piacenza, Italy); Cristian Dalla Fiora, Claudio Fattibene, Maria Michiara, and Paolo Orsi (Local Health Authority, Parma, Italy); Paolo Giorgi Rossi and Lucia Mangone (Epidemiology Unit, Azienda Unità Sanitaria Locale, IRCCS di Reggio Emilia, Reggio Emilia, Italy); Cinzia Campari and Romano Sassatelli (Cancer Screening Unit, Azienda Unità Sanitaria Locale, IRCCS di Reggio Emilia, Reggio Emilia, Italy); Giuliano Carrozzi, Federica Rossi, Pasqualina Esposito, Paolo Trande, and Simona Viani (Local Health Authority, Modena, Italy), Luigi Ricciardiello, Vincenzo Cennamo, Giovanna Gualandi, and Francesca Mezzetti (Local Health Authority, Bologna, Italy); Licia Caprara and Margherita De Lillo (Local Health Authority, Imola, Italy); Aldo De Togni, Caterina Palmonari, Daniela Pasquali, and Giorgio Zoli (Local Health Authority, Ferrara, Italy); Serena Dal Re, Chiara Petrini, Monica Serafini, Omero Triossi, and Benedetta Vitali (Local Health Authority, Ravenna, Italy); Mara Gallinucci, Claudia Imolesi, Mauro Palazzi, and Carlo Fabbri (Local Health Authority, Cesena, Italy); and Debora Canuti, Coralba Casale, Mauro Giovanardi, and Daniele Trombetti (Local Health Authority, Rimini, Italy). 